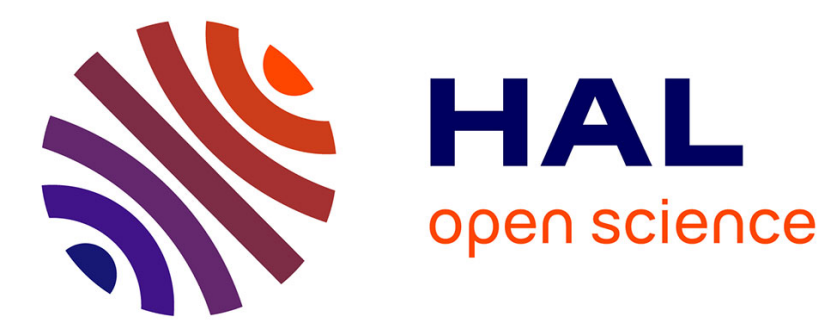

\title{
Time reversal of photoacoustic waves
}

Emmanuel Bossy, Khalid Daoudi, Albert-Claude Boccara, Mickaël Tanter, Jean-François Aubry, Gabriel Montaldo, Mathias Fink

\section{To cite this version:}

Emmanuel Bossy, Khalid Daoudi, Albert-Claude Boccara, Mickaël Tanter, Jean-François Aubry, et al.. Time reversal of photoacoustic waves. Applied Physics Letters, 2006, 89 (18), pp.184108. $10.1063 / 1.2382732$. hal-02481756

\section{HAL Id: hal-02481756 https://hal.science/hal-02481756}

Submitted on 17 Feb 2020

HAL is a multi-disciplinary open access archive for the deposit and dissemination of scientific research documents, whether they are published or not. The documents may come from teaching and research institutions in France or abroad, or from public or private research centers.
L'archive ouverte pluridisciplinaire HAL, est destinée au dépôt et à la diffusion de documents scientifiques de niveau recherche, publiés ou non, émanant des établissements d'enseignement et de recherche français ou étrangers, des laboratoires publics ou privés. 


\section{Time reversal of photoacoustic waves}

Cite as: Appl. Phys. Lett. 89, 184108 (2006); https://doi.org/10.1063/1.2382732

Submitted: 13 July 2006 . Accepted: 18 September 2006 . Published Online: 03 November 2006

Emmanuel Bossy, Khalid Daoudi, Albert-Claude Boccara, Mickael Tanter, Jean-François Aubry, Gabriel Montaldo, and Mathias Fink

\section{ARTICLES YOU MAY BE INTERESTED IN}

Photoacoustic imaging in biomedicine

Review of Scientific Instruments 77, 041101 (2006); https://doi.org/10.1063/1.2195024

Photoacoustic guidance of high intensity focused ultrasound with selective optical contrasts and time-reversal

Applied Physics Letters 94, 054102 (2009); https://doi.org/10.1063/1.3077018

Iterative reconstruction algorithm for optoacoustic imaging

The Journal of the Acoustical Society of America 112, 1536 (2002); https://

doi.org/10.1121/1.1501898

Lock-in Amplifiers Find out more today

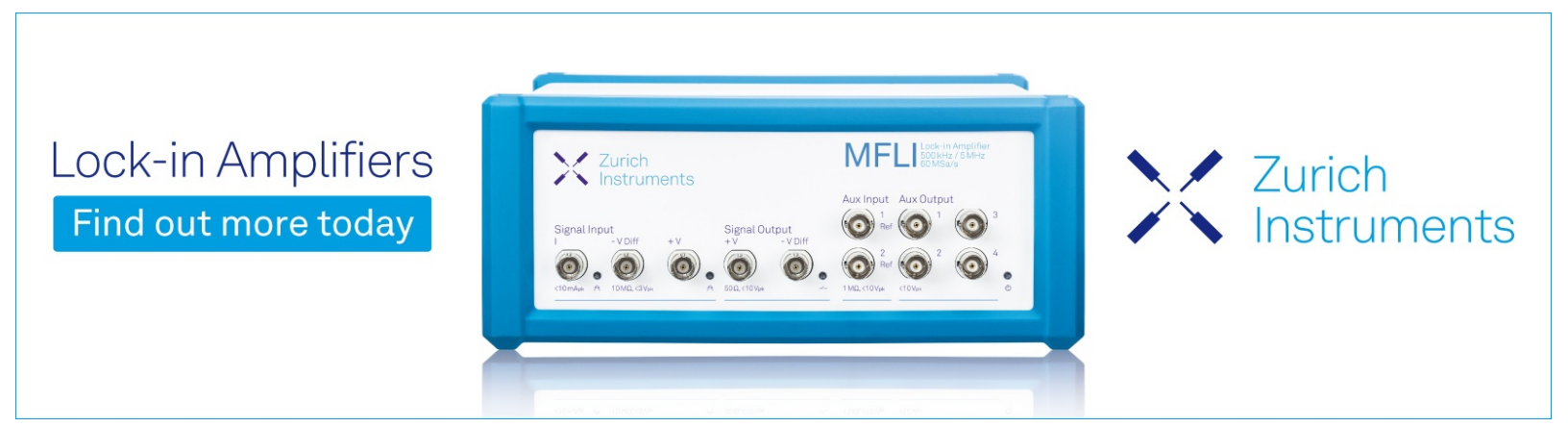




\title{
Time reversal of photoacoustic waves
}

\author{
Emmanuel Bossy, ${ }^{\text {a) }}$ Khalid Daoudi, and Albert-Claude Boccara \\ Laboratoire d'Optique Physique, ESPCI, CNRS UPR 5, 10 Rue Vauquelin, 75231 Paris Cedex 05, France
}

Mickael Tanter, Jean-François Aubry, Gabriel Montaldo, and Mathias Fink

Laboratoire Ondes et Acoustique, ESPCI, Université Paris VII, CNRS UMR 7587, 10 Rue Vauquelin, 75231

Paris Cedex 05, France

(Received 13 July 2006; accepted 18 September 2006; published online 3 November 2006)

\begin{abstract}
In this work, the authors use the photoacoustic effect to create a source for ultrasonic time-reversal experiments. Photoacoustic waves were generated by an optically absorbing gel sphere excited by a laser pulse in a highly optically diffusive solution and recorded with an ultrasound array controlled by a time-reversal electronics. The emission of the time-reversed photoacoustic waves allowed the refocusing of the ultrasound towards the optical absorber, in particular, in the presence of a strongly acoustically defocusing medium. The authors illustrate how the technique can be applied to perform acoustical imaging in the presence of a strong acoustical aberration. () 2006 American Institute of Physics. [DOI: 10.1063/1.2382732]
\end{abstract}

For more than a decade, time-reversal techniques have been developed in many different fields of applications including the detection of defects in solids, underwater acoustics, room acoustics, and medical imaging and therapy. ${ }^{1,2}$ The essential property that makes time-reversed acoustics possible is that the underlying physical process of wave propagation remains unchanged when time is reversed. In a nondissipative medium, the equations governing the waves guarantee that for every burst of sound that diverges from a source, there is in theory a set of waves that can precisely retrace the path of the sound back to the source. This reference source can be generated by introducing an active source in the desired focal area. In the medical field of lithotripsy, an echo backscattered by a strongly reflecting target (either the kidney stone or gallstone) is used as the reference signal for the time-reversed destructing ultrasonic beam. ${ }^{2}$ In that case, time reversal permits us to correct both for aberrations occurring from medium heterogeneities and for respiratory motion artifacts. However, in many practical situations it is not possible to insert an active source in the medium nor to rely on the presence of a unique strong scatterer. In analogy with adaptive optics in astronomy, it is nevertheless possible to create artificial "ultrasonic stars" in the body. A first approach was recently proposed that consists of inducing a cavitation bubble using an initial focused ultrasound beam in the desired focal area. ${ }^{3}$ The bubble cavitation generates a spherical wave that can be used as a reference for a second corrected illumination using the time-reversal principle. In this letter, we propose an approach enabling the remote creation of ultrasonic stars in the body without cavitation. We make use of the photoacoustic effect in order to generate, time reverse, and refocus ultrasound towards an optical contrast.

Optically absorbing regions may indeed act as acoustic sources through the photoacoustic effect described by the following equation:



$$
\Delta p-\frac{1}{c^{2}} \frac{\partial^{2} p}{\partial t^{2}}=-\frac{\beta}{c_{p}} \frac{\partial H}{\partial t},
$$

where $p$ is the acoustic pressure, $c$ the speed of sound, $\beta$ the isobaric thermal expansion coefficient, $c_{p}$ the specific heat, and $H$ the volumetric density of optical energy per unit time. Physically, photoacoustic waves are therefore pressure waves generated by the thermoelastic expansion that follows the absorption of a light pulse. ${ }^{4,5}$ This effect is at the origin of recently developed photoacoustic imaging methods, which use passive ultrasonic detection to reconstruct maps of the optical absorption. ${ }^{6-8}$ In biological tissues, which are generally strong optically scattering media hindering conventional optical imaging based on ballistic light, these methods provide images of optical absorption with the ultrasonic resolution. In this work, we experimentally perform time reversal of photoacoustic waves generated by an optical absorber embedded in a highly optically diffusive medium.

A schematic diagram of the proof-of-principle experimental setup is shown in Fig. 1. A frequency-doubled $Q$-switched Nd:YAG laser $(\lambda=532 \mathrm{~nm}$, Brilliant, Quantel, France) was used to deliver $5 \mathrm{~ns}$ light pulses with an energy of approximately $80 \mathrm{~mJ}$ per pulse, with a pulse repetition frequency of $10 \mathrm{~Hz}$. A 64-element ultrasound array with a



FIG. 1. Schematic diagram of the experimental setup. LB: laser beam, DV: divergent lens, DL: diffusive light, AS: absorbing sphere, HGL: hydrophone, TS: translation stage, IL: Intralipid solution, UA: ultrasound array, and RS: rubber slab. 

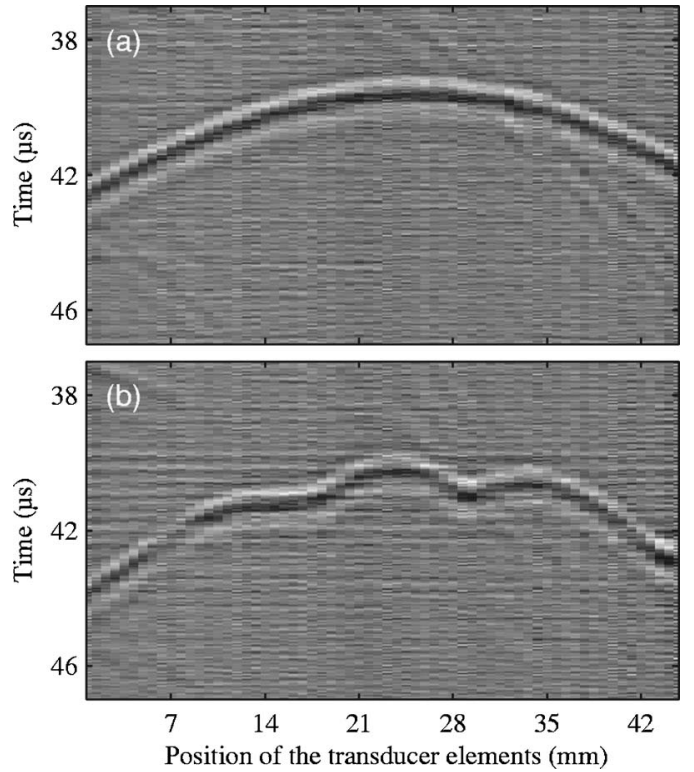

FIG. 2. Photoacoustic signals (vertical dimension) measured on the 64element ultrasound array (horizontal dimension). (a) Photoacoustic wavefront detected after propagation in the Intralipid solution. (b) Photoacoustic wavefront detected in the presence of a strongly defocusing rubber slab pressed against the ultrasound array.

central frequency of $1.5 \mathrm{MHz}$ was immersed in a tank and connected to a 64-channel time-reversal electronics (Lecoeur Electronique, Chuelles, France). The array dimension is $10 \mathrm{~mm}$ along the $y$ axis and has an aperture of approximately $45 \mathrm{~mm}$ (interelement pitch of $0.7 \mathrm{~mm}$ along the $x$ axis). The 64 independent channels allow us to sample the ultrasonic signals on the receive mode (sampling frequency of $80 \mathrm{MHz}$ ) and to emit a fully programmable voltage on the transmit mode. The ultrasound acquisition was triggered using the trigger output of the laser $Q$ switch. A 400- $\mu \mathrm{m}$-diameter hydrophone (HGL-400, Onda Corporation, Sunnyvale, CA), mounted on computer-controlled $X Y Z$ translation stages, was used to map the ultrasonic field. A standard personal computer was used to control both the electronics and the translation stages and to process the ultrasonic data. The tank was filled with a $0.4 \%$ Intralipid solution (obtained by diluting 50 times a stock solution of $20 \%$ Intralipid) to provide a highly optically scattering medium ${ }^{9}$ with a reduced scattering coefficient $\mu_{s} \approx 6 \mathrm{~cm}^{-1}$. Prior to entering the Intralipid solution, the laser beam was expanded to a diameter of the order of $2 \mathrm{~cm}$ to minimize parasitic photoacoustic emission created at the surface. A transparent alginate gel sphere, dyed with India ink to obtain an absorption coefficient $\mu_{a} \approx 10 \mathrm{~cm}^{-1}$, was used as the optical absorber. The diameter of the sphere, approximately $1 \mathrm{~mm}$, was chosen to match the main frequency components of the photoacoustic wave to the frequency bandwidth of the transducer. ${ }^{5}$ The sphere was fabricated directly on a 100 - $\mu$ m-diameter nylon thread, which was also used to hang and position the sphere in the imaging plane. The sphere was hung into the solution at a location corresponding to a depth of approximately $3 \mathrm{~cm}$ relative to the penetration of light $(X$ direction). The distance from the sphere to the transducer array was approximately $6 \mathrm{~cm}$ ( $Z$ direction). A Rubber slab with heterogeneous thickness (varying from approximately 3 to $6 \mathrm{~mm}$ ) was placed against the ultrasound array to provide strong ultrasonic phase aberration. The ultrasound ve-



FIG. 3. Maximum signal amplitude detected with a hydrophone, as a function of the hydrophone position across the focal plane. (-) Focal spot generated by time reversing the photoacoustic waves measured in the presence of the defocusing rubber slab. (--) Focal spot generated by time reversing the photoacoustic waves measured without defocusing slab. ( $\cdots$ ) Focal spot generated by time reversing the photoacoustic signal measured without defocusing slab, but time reversed in the presence of the slab.

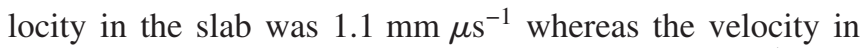

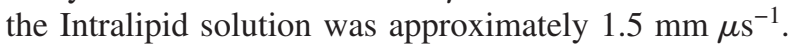

First, photoacoustic signals generated by the absorbing sphere were measured on the 64-element array, with and without the aberrating slab. Figure 2 shows the two corresponding wavefronts. Signals were coherently time averaged $(n=100)$ to improve signal-to-noise ratio. Figure 2(b) clearly shows the wavefront distortion introduced by the aberrating slab. For each case, with and without the aberrating slab, the measured signals were time reversed and reemitted by the array. To assess the quality of the focusing, the hydrophone was linearly scanned along the $X$ direction across the location of the sphere after the sphere had been removed. Figure 3 plots the normalized pressure peak amplitude recorded on the hydrophone and therefore illustrates the quality of the focusing. The focal spot obtained in the presence of the defocusing rubber slab (solid line) turns out to be nearly identical to that obtained without the rubber slab (dashed line), which demonstrates that time reversing the photoacoustic wave allows us to refocus with the same quality as that obtained in an acoustically homogeneous medium. To illustrate the efficiency of the time-reversal process, Fig. 3 also plots the focal spot measured in the presence of the aberrating medium when focusing was performed assuming the medium to be acoustically homogeneous (dotted line).

Time reversal of photoacoustic waves have several potential applications in the context of imaging optically diffu-

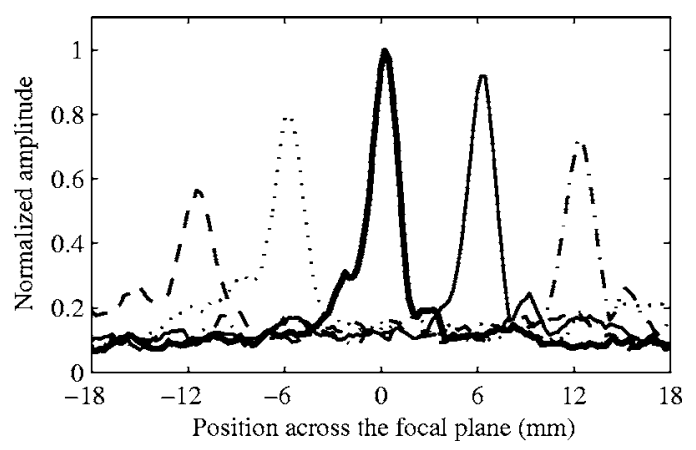

FIG. 4. Focal spots measured in the presence of the defocusing slab, generated by time reversing the photoacoustic waves after linear delay laws were added to the signals. The five focal spots correspond to five different delay laws based on propagation in a homogeneous medium, calculated in order to focus at $-12,-6,0,+6$, and $+12 \mathrm{~mm}$. 




FIG. 5. $B$ mode ultrasound images of two nylon wires, obtained in the presence of the defocusing rubber slab pressed against the transducer array. Image (a) was obtained without taking into account the presence of the slab. Image (b) was obtained by time reversing the photoacoustic waves measured in the presence of the defocusing slab. The optically absorbing sphere was located on the nylon wire located at $x=-3 \mathrm{~mm}$.

sive media. First, a purely optical contrast with no acoustic contrast is not detectable in tissues by conventional ultrasound, whereas the emitted photoacoustic waves can reveal the presence of the absorber. Furthermore, time reversing the photoacoustic signals and convolving them with long highintensity sine bursts could allow us to perform high-intensity focused ultrasound on targets of optical nature, even if the optical contrast is embedded in an acoustically aberrating medium. Second, a purely optical contrast can be used to focus into an acoustically aberrating medium with the aim of acoustically imaging this medium. We now illustrate this second example in further detail. A second nylon wire was hung about $8 \mathrm{~mm}$ away (along the $X$ direction) from the nylon wire supporting the absorbing sphere. The two wires were acoustically equivalent objects, as the acoustic scattering from the optically absorbing gel sphere was negligible compared to that from the wires. Our objective was to show that time reversal of photoacoustic waves allows us to acoustically image the two wires even when these are located behind a highly acoustically aberrating media. The results above show that it is possible to focus toward the optical contrast. However, in order to perform an image, focusing needs to be performed at different locations. While time reversal only allows us to refocus at the location of the initial source, it is nevertheless possible to steer the beam by delaying the time-reversed signals with a linear time-delay law, as would be done for a homogeneous media. In this case, the ultrasonic beam will remain reasonably focused after steer- ing within a limited region around the original focal point, defined as the isoplanetic region. . $^{10,11}$

Figure 4 plots the focal spots obtained for different steering angles and shows that under our experimental conditions the isoplanetic region extends over several focal spot widths. In order to image acoustical contrasts located within the isoplanetic region, it is also necessary to beamform the received signals, as was done to the transmitted signals. As for focusing on the transmit mode, the appropriate time-delay and amplitude laws needed to perform beamforming on the receive mode is not explicitly known. Nevertheless, as the focusing laws are strictly identical for transmit and receive modes, beamforming on the receive mode can be simply obtained by time convolving the received wavefront with the transmitted wavefront and summing the results over all the channels. ${ }^{11}$ Figure 5 shows two acoustical images obtained with this procedure, with the aberrator pressed against the transducer. Figure 5(a) shows the image obtained in the presence of the slab with standard beamforming, assuming no aberrator. The poor quality of the image further illustrates the strong defocusing effect of the rubber slab. On the other hand, Fig. 5(b) shows the image based on the time-reversed photoacoustic signal, on which the two wires are very clearly distinguished. In this example, the photoacoustic signals were therefore used as a way to learn how to focus in the vicinity of the optical contrast and not to build a photoacoustic image (which requires the absence of acoustic aberrations). The dimension of the image is only limited by the extent of the isoplanetic region. Imaging a larger area would require the existence of several localized optical contrasts under the condition that their associated photoacoustic signals could be discriminated before selectively time reversed.

In conclusion, localized optical absorption can act as acoustic sources, which can be used as primary sources for ultrasound time-reversal techniques. As for photoacoustic imaging techniques, this technique is only limited by the amount of light that may reach optical absorbers regardless of the propagation of the light (diffusive or ballistic). In the biomedical field, the feasibility of such a technique will depend on the existence of well localized optical absorbers or on the ability to produce such absorbers (using exogenous contrast agents, for instance).

${ }^{1}$ M. Fink, D. Cassereau, A. Derode, C. Prada, P. Roux, M. Tanter, J. L. Thomas, and F. Wu, Rep. Prog. Phys. 63, 1933 (2000).

${ }^{2}$ M. Fink, G. Montaldo, and M. Tanter, Annu. Rev. Biomed. Eng. 5, 465 (2003).

${ }^{3}$ M. Pernot, G. Montaldo, M. Tanter, and M. Fink, Appl. Phys. Lett. 88 (2006).

${ }^{4}$ G. J. Diebold and T. Sun, Acustica 80, 339 (1994).

${ }^{5}$ V. G. Andreev, A. A. Karabutov, and A. A. Oraevsky, IEEE Trans. Ultrason. Ferroelectr. Freq. Control 50, 1383 (2003).

${ }^{6}$ G. Ku, X. Wang, X. Xie, G. Stoica, and L. V. Wang, Appl. Opt. 44, 770 (2005).

${ }^{7}$ M. Xu and L. V. Wang, Med. Phys. 29, 1661 (2002).

${ }^{8}$ S. Manohar, A. Kharine, J. C. van Hespen, W. Steenbergen, and T. G. van Leeuwen, Phys. Med. Biol. 50, 2543 (2005).

${ }^{9}$ H. J. Vanstaveren, C. J. M. Moes, J. Vanmarle, S. A. Prahl, and M. J. C. Vangemert, Appl. Opt. 30, 4507 (1991).

${ }^{10}$ M. Tanter, J. L. Thomas, and M. Fink, J. Acoust. Soc. Am. 103, 2403 (1998).

${ }^{11}$ C. Dorme and M. A. Fink, IEEE Trans. Ultrason. Ferroelectr. Freq. Control 43, 167 (1996). 